\title{
Identification of Leaf Rust Resistance Genes in Bread Wheat Cultivars from Ethiopia
}

\author{
Takele Weldu Gebrewahid, ${ }^{1,2}$ Pei-pei Zhang, ${ }^{1}$ Zhan-jun Yao, ${ }^{3}$ Zai-feng Li, ${ }^{1, \dagger}$ and Da-qun Liu ${ }^{1, \dagger}$ \\ ${ }^{1}$ College of Plant Protection, Hebei Agricultural University, Baoding, Hebei 071001, China \\ ${ }^{2}$ College of Agriculture, Aksum University, Shire-Indaslassie, Tigray 314, Ethiopia \\ ${ }^{3}$ College of Agronomy, Agricultural University of Hebei/North China Key Laboratory for Crop Germplasm Resources, Baoding, \\ Hebei 071001, China
}

\begin{abstract}
Wheat leaf rust, caused by Puccinia triticina ( $P t)$, is a widespread disease of bread wheat worldwide. In the present study, 50 wheat cultivars from Ethiopia and 34 differential lines, mostly near-isogenic lines (NILs) in the background of Thatcher with known resistance genes to leaf rust $(L r)$, were tested with $14 P t$ races in the greenhouse to postulate $L r$ genes at the seedling stage. Field experiments were also conducted to identify adult plant responses to leaf rust in Baoding in the 2017-2018 and 2018-2019 growing seasons and in Zhoukou in the 2018-2019 growing season. Thirteen $L r$ genes (Lr1, Lr18, Lr3ka, Lr15, Lr26, Lr20, Lr14a,

12 cultivars were the most commonly identified resistance genes. Less frequently identified genes included $\operatorname{Lr} 26$ (five cultivars); $\operatorname{Lr} 30$ and $L r 18$ (each present in four cultivars); $L r 15, L r 3 k a$, and $L r 2 a$ (each identified in three cultivars); and $L r 68$ (two cultivars). Evidence for the existence of $L r 11, L r 20$, and $L r 14 a$ (each in one cultivar) was also obtained. Twenty-one cultivars were found to have slow rusting resistance to leaf rust in the field tests. The results should be valuable for cultivar selection with combinations of effective $L r$ genes and used in breeding new cultivars with improved resistance to leaf rust in Ethiopia and China.
\end{abstract} $L r 30, L r 2 a, L r 11, L r 34, L r 46$, and $L r 68$ ) either singly or in combination were found in 39 cultivars. Known $L r$ genes were not present in the remaining 11 cultivars. $L r l$ and $L r 46$, each in 13 cultivars, and $L r 34$ in
Keywords: wheat leaf rust, gene postulation, adult-plant resistance, molecular marker
Wheat (Triticum aestivum L.) is the second major food crop next to rice produced worldwide. Ethiopia is the most important wheat growing country in sub-Saharan Africa with about 1.7 million hectares and $2.7 \mathrm{t}$ grain yield/ha productivity (CSA 2017). Wheat is ranked fourth next to teff (Eragrostis tef [Zucc.] Trotter), maize (Zea mays L.), and sorghum (Sorghum bicolor [L.] Moench) in terms of production in Ethiopia (CSA 2017). However, wheat production in Ethiopia is disposed to diverse biotic and abiotic stresses, among which rusts are most important.

Leaf rust, caused by the fungus Puccinia triticina Erikss. (Pt), is an important disease of wheat in Ethiopia (Badebo et al. 2008; Hei 2017). The potential yield loss for leaf rust-susceptible cultivars can be more than $60 \%$ (Chen 2014). Resistant cultivars are considered the most cost-effective and eco-friendly approach to control leaf rust. Genetic resistance to wheat rust pathogens is usually categorized as either race-specific seedling resistance or race-non-specific resistance (Chen 2013). Race-specific resistance is usually controlled by major genes and is effective against some races of the pathogen with corresponding avirulence genes (Flor 1942). On the other hand, race-non-specific resistance is considered as a broad spectrum effective against all races of the pathogen. Race-non-specific resistance is considered as more durable and generally expressed at the adult plant stage governed by a number of minor-effect resistance genes. This

${ }^{\dagger}$ Corresponding authors: Z. F. Li; lzf7551@aliyun.com and D. Q. Liu; 1dq@hebau.edu.cn

Funding: This study was supported by National Key Research and Development Plan (2017YFD0300906-07).

*The $\boldsymbol{e}$-Xtra logo stands for "electronic extra" and indicates that one supplementary table is published online.

The author(s) declare no conflict of interest.

Accepted for publication 28 March 2020.

(C) 2020 The American Phytopathological Society type of resistance is commonly called slow rusting or adult plant resistance (APR) (Caldwell 1968).

Identification of leaf rust resistance $(\mathrm{Lr})$ genes existing in the released wheat cultivars helps in appropriate cultivar deployment to manage the disease. Multirace testing and molecular marker genotyping are useful approaches to gene postulation to understand the $\mathrm{Lr}$ genes present in wheat cultivars.

Up to now, about $79 \mathrm{Lr}$ genes have been reported in wheat (McIntosh et al. 2017). Most of the reported $L r$ genes are race-specific conferring hypersensitive reactions and interact with the pathogen in a gene-forgene manner (Flor 1956). However, Lr34 (Lagudah et al. 2006), Lr46 (Singh et al. 1998), $\operatorname{Lr67}$ (Herrera-Foessel et al. 2011), and Lr68 (Herrera-Foessel et al. 2012) confer slow rusting resistance despite a susceptible reaction.

The gene-for-gene hypothesis by Flor (1956) has been used by researchers to identify $L r$ genes present in wheat cultivars. For example, Gao et al. (2019) reported nine $L r$ genes ( $L r 2 a, L r 3 k a, L r 1$, $L r 30, L r 26, L r 15, L r 36, L r 37$, and $L r 46)$ in 86 wheat cultivars using $14 P t$ races. Gebrewahid et al. (2017) postulated $12 \mathrm{Lr}$ genes (Lr3ka, Lr11, Lr10, Lr2b, Lr1, Lr26, Lr13, Lr21, Lr34, Lr37, $L r 44$, and $L r 46$ ) from 83 wheat cultivars using $18 P t$ races. Fourteen Lr genes (Lr2a, Lr3bg, Lr3ka, Lr14a, Lr1, Lr16, Lr17a, Lr23, $L r 24, L r 26, L r 18, L r 20, L r 34$, and $L r Z H 84)$ were identified in 102 wheat cultivars using $24 P t$ races (Li et al. 2010). Mebrate et al. (2008) stated that $18 \mathrm{Lr}$ genes $(L r 2 c, L r 3, L r 3 k a, L r 9, L r 10$, Lr14a, Lr14b, Lr1, Lr13, Lr20, Lr21, Lr23, Lr27+31, Lr16, Lr18, Lr30, Lr37, and Lr44) were identified in 36 wheat cultivars using $31 P t$ races. Pathan and Park (2006) reported Lr3a, Lrl, Lr17b, Lr10, Lr3ka, Lr13, Lr20, Lr26, Lr37, and Lr14a in 105 cultivars using five $P t$ races. Maaroof et al. (2005) reported $L r 13$, $L r 16, L r 1, L r 3, L r 10, L r 23, L r 17$, and $L r 26$ in 22 wheat cultivars with $13 P t$ races. Lr3bg, Lr14a, Lr1, Lr3, Lr10, Lr16, Lr13, $L r 23$, and $L r 26$ were reported in 163 wheat cultivars using $14 P t$ races (Singh et al. 1999).

Molecular markers linked to some $L r$ genes have been developed to detect specific genes. For instance, $\operatorname{Lrl}$ (Cloutier et al. 2007), $\operatorname{Lr} 9$ (Schachermayr et al. 1994), Lr10 (Schachermayr et al. 1997), Lr19 (Gupta et al. 2005), Lr20 (Neu et al. 2002), Lr24 (Schachermayr et al. 1995), Lr26 (Chai et al. 2006), Lr37 (Helguera et al. 2003), 
Lr46 (Singh et al. 1998), Lr67 (Herrera-Foessel et al. 2011), and Lr68 (Herrera-Foessel et al. 2012) can be identified using closely linked molecular markers. Allele-specific markers for APR genes like Lr34 (Lagudah et al. 2009) have been developed and used for marker-based detection.

In Ethiopia, information on $\mathrm{Lr}$ genes in released wheat cultivars is limited, which makes it difficult to exploit resistant cultivars for managing leaf rust. Ethiopia is a hot spot for three wheat rusts: stem, stripe, and leaf rusts. Therefore, identifying $L r$ genes in the presently grown wheat cultivars is enormously useful for managing leaf rust using gene deployment schemes and for breeding new resistant cultivars. Hence, the objective of this study was to identify $L r$ genes in 50 bread wheat cultivars from Ethiopia.

\section{Materials and Methods}

Plant materials and $\boldsymbol{P t}$ races. Fifty wheat cultivars from Ethiopia and 34 differential lines, mostly near-isogenic lines (NILs) in the background of Thatcher with known $L r$ genes kindly provided by CIMMYT, were used to test at the seedling stage in the greenhouse to $14 P t$ races collected from China (Tables 1, 2, and 3). Fresh urediniospores of $P t$ races from the Department of Plant Pathology, Hebei Agricultural University, China, were obtained by multiplying urediniospores on the susceptible cultivar Zhengzhou 5389 in a greenhouse. These $P t$ races were named following the Puccinia recondita f. sp. tritici code system described by Long and Kolmer (1989), with adding a fourth letter for the reactions to the fourth quartet of differentials (http://www.ars.usda.gov/SP2UserFiles/ad_hoc/36400500Cereal

Table 1. The pedigree and origin of Ethiopian bread wheat cultivars tested for leaf rust response ${ }^{a}$

\begin{tabular}{|c|c|c|c|}
\hline No. & Cultivar & Origin & Pedigree \\
\hline 1 & Abola & CIMMYT/Ethiopia & BOBWHITE/BUCKBUCK \\
\hline 2 & Alidoro & Ethiopia/U.S.A. & HK-14-R251 \\
\hline 3 & Biqa & NA & NA \\
\hline 4 & Bobicho & CIMMYT & PEREGRINE/PF-70354/4/KALYANSONA/BLUEBIRD//ALONDRA/3/MARINGA \\
\hline 5 & Bolo & CIMMYT & VEE/LIRA//BOW/3/BCN/4/KAUZ \\
\hline 6 & Dandaa & CIMMYT & KIRITATI//2*PBW-65/2*SERI-82 \\
\hline 7 & Dereselgn & CIMMYT & 300-SM-501-M/HAR-1709 \\
\hline 8 & Dashen & CIMMYT & VEE17KVZ/BUHO“S”//KAL-BB \\
\hline 9 & Digelu & CIMMYT & SHANGHAI-7/KAUZ,MEX \\
\hline 10 & Dinknesh & CIMMYT & CARACARA/4/CORYDON/3/PELOTAS-72380/ARTHUR-71*2/H-567-1 \\
\hline 11 & Dure & CIMMYT & BOBWHITE/YDING//ZENZONTLI \\
\hline 12 & Enkoy & Kenya & HEBRARD//WISCONSIN245/SUPREMO51/3/2*FROCOR/FRONTANA//YAQUI \\
\hline 13 & ET-13A2 & Ethiopia & UQ105 × ENKOY \\
\hline 14 & Galil & Israel & HORK/YAMHILL//KALYANSONA/BLUEBIRD/3/BOBWHITE \\
\hline 15 & Gambo & CIMMYT & AVRORA//KALYANSONA/BLUEBIRD/3/(SIB)WOODPECKER \\
\hline 16 & Gassy & CIMMYT & PFAU/SERI//BOBWHITE \\
\hline 17 & Hawi & CIMMYT/Ethiopia & CHILERO/PARULA \\
\hline 18 & Hidasse & CIMMYT/Ethiopia & $\begin{array}{l}\text { YANAC/3/PARULA/ICTA-SARA-82//TESIA-79/VEERY-5/4/CROC-1/(224) } \\
\text { AE.SQ//OPATA }\end{array}$ \\
\hline 19 & Hobora & NA & NA \\
\hline 20 & Hogona & NA & PYN/BAU//MILLAN \\
\hline 21 & Huluka & ICARDA/Ethiopia & UTIQUE-96/3/PAYNE/BAGULA//MILAN \\
\hline 22 & K6295-4A & Kenya & ROMANY $\times$ GB-GAMENYA \\
\hline 23 & Kakaba & CIMMYT & KIRITATI//SERI-82/RAYON-89 \\
\hline 24 & KBG-01 & Ethiopia/Germany & 300-SM-501-M/HAR-1709 \\
\hline 25 & Katar & CIMMYT & $\mathrm{COOK} / \mathrm{VEE} / / \mathrm{DOVE} / \mathrm{SERI} / 3 / \mathrm{BJY} / \mathrm{COC}$ \\
\hline 26 & Kingbrid & NA & NA \\
\hline 27 & Kubsa & CIMMYT & NORD-DESPREZ/VG-9144//KALYANSONA/BLUEBIRD/3/YACO/4/VEERY-5 \\
\hline 28 & Kulkulu & ICARDA & PYN/BAU//MILAN \\
\hline 29 & Lakech & NA & NA \\
\hline 30 & Lemu & NA & NA \\
\hline 31 & Mandoyu & CIMMYT & WORRAKATTA/PASTOR \\
\hline 32 & Mekelle-01 & NA & CPAN-1962/TONICHI-81//LIRA/PARULA \\
\hline 33 & Mekelle-02 & NA & HI-999/HI-601 \\
\hline 34 & Mekelle-03 & NA & NA \\
\hline 35 & Mekelle-04 & NA & NA \\
\hline 36 & Millennium & CIMMYT & ALONDRA/CEP 75630//CEP 75234/PAT 7219/3/BUCKBUCK/BIY/4/ \\
\hline 37 & Mitike & Ethiopia & BOBWHITE/ROMANY-BC \\
\hline 38 & Monqolle & NA & NA \\
\hline 39 & Ogalcho & CIMMYT & WORRAKATTA/2*PASTOR \\
\hline 40 & Pavon 76 & CIMMYT & VICAM-71//CIANO-67/SIETE-CERROS-66/3/KALYANSONA/BLUEBIRD \\
\hline 41 & Shorima & ICARDA & UTIQUE-96/3/PAYNE/BAGULA//MILAN \\
\hline 42 & Simba & CIMMYT & PARULA/VEERY-6//MYNA/VULTURE \\
\hline 43 & Sirbo & CIMMYT & VS73.600/MRL/3/BOW//YR/TRF \\
\hline 44 & Suf-Omer & CIMMYT & 4777*2/4/FRONTANA/KENYA-58//NEWTHATCH/3/(GB)GABO/5/PAVON-76 \\
\hline 45 & Sula & CIMMYT & MAIORAL(SIB)/BUCKBUCK(HAR-7140)//REIHENBACHII \\
\hline 46 & Tay & NA & NA \\
\hline 47 & Tossa & CIMMYT & ATTILA \\
\hline 48 & Tsehay & NA & VEERY-5/ICTA-SARA-82//DUCULA \\
\hline 49 & Tusie & CIMMYT & COOK/VEE“S”//DOVE“S”/SERI \\
\hline 50 & Wabe & NA & MRL“S”-BUC“S” \\
\hline
\end{tabular}

${ }^{\text {a }} \mathrm{NA}=$ not available. 
rusts/pt_nomen.pdf). Infection types (ITs) exhibited by the cultivars and NILs at the seedling tests were used for postulating $L r$ genes (Tables 2 and 3).

Seedling testing. Thirty-four NILs, 50 wheat cultivars, and Zhengzhou 5389 were grown in a plastic growth chamber $(4 \times$ $3 \mathrm{~cm}$ ) in the greenhouse and inoculated with $14 P t$ races (TGPQ, FHJQ, PHTS, KHSS, FGSS, KHSQ, FHNQ, KHJS, FHSQ, FHTQ, FGSD, FGGS, FGSR, and PHJT) (Tables 2 and 3). Inoculation at the seedling stage was done by brushing urediniospores from sporulating Zhengzhou 5389 seedlings onto test seedlings when the first leaves were fully expanded. Inoculated seedlings were then placed in plastic-covered cages and incubated at $18^{\circ} \mathrm{C}$ and $100 \%$ relative humidity (RH) for about $24 \mathrm{~h}$. They were then placed into a growth chamber with $12 \mathrm{~h} \mathrm{light} / 12 \mathrm{~h}$ darkness at 18 to $20^{\circ} \mathrm{C}$ with $70 \%$ RH. ITs were scored based on the 0 to 4 scale described by Roelfs et al. (1992) 14 days postinoculation. The gene postulation was then done based on the method described by Dubin et al. (1989).

Field testing. All the wheat cultivars, including Saar (resistant control) and Zhengzhou 5389 (susceptible control), were planted in the field at Baoding (Hebei province) in the 2017-2018 and 2018-2019 growing seasons, and at Zhoukou (Henan province) in the 2018-2019 growing season for evaluating leaf rust reaction. Field experiments were managed in randomized complete blocks replicated twice. About 60 to 70 seeds of each cultivar were sown in a row with $1.5-\mathrm{m}$ length and $30 \mathrm{~cm}$ between rows. Zhengzhou 5389 was grown perpendicularly and adjacently to the test rows to help spread of spores within the experiments. Races TGPQ, PHTS, and PHJT were selected for field inoculation based on their wider virulence on the wheat varieties in the seedling test than other races.
Equal amounts of urediniospores from TGPQ, PHTS, and PHJT were mixed and suspended in $0.03 \%$ Tween 20 and were then inoculated onto spreader rows at the tillering stage. One-month postinoculation, maximum disease severity (MDS) was recorded using the modified percentage scale described by Cobb (1948) when Zhengzhou 5389 had $90 \%$ disease severity.

Molecular marker detection. Genomic DNA of all 50 wheat cultivars was extracted using the cetyl trimethylammonium bromide method (Gill et al. 1991). Molecular markers closely linked or allele-specific to 12 known genes ( $\operatorname{Lrl}, \operatorname{Lr} 9, \operatorname{Lr} 10, \operatorname{Lr} 19, \operatorname{Lr} 20$, Lr24, Lr26, Lr34, Lr37, Lr46, Lr67, and Lr68) were used to test all cultivars. Polymerase chain reaction (PCR) was conducted according to the technique described by Helguera et al. (2003). A total of $20 \mu \mathrm{l}$ of reaction mixture contained $2 \mu \mathrm{l}(4 \mathrm{ng} / \mu \mathrm{l})$ of template DNA, $2 \mu \mathrm{l}(4 \mathrm{~mol} / \mu \mathrm{l})$ of primer, $6 \mu \mathrm{l}$ of $\mathrm{ddH}_{2} \mathrm{O}$, and $10 \mu \mathrm{l}$ of $2 \times \mathrm{Taq}$ PCR Master Mix (Tiangen Biochemical Incorporation, Beijing, China). All PCR annealing temperatures, allele sizes, and primer sequences are provided in Table 4 . The amplified products were separated by $1.5 \%$ agarose gel electrophoresis for all of the markers except $12 \%$ nondenaturing polyacrylamide gel electrophoresis for the cleaved amplified polymorphic sequence (CAPS) marker csLV46G22 specific to Lr46.

Statistical analysis. Analysis of variance was performed using the generalized linear model procedure (PROC GLMMIX) of Statistical Analysis System (version 9.1; SAS Institute, Cary, NC). Environments (the combination of location and year), cultivars, and their interaction were taken as fixed effects, and replications nested in environments were taken as random. Fisher's least significant difference test at $P<0.05$ was used to separate the treatment of MDS

Table 2. Infection types ${ }^{\mathrm{a}}$ of 34 tester lines inoculated with 14 Puccinia triticina races

\begin{tabular}{|c|c|c|c|c|c|c|c|c|c|c|c|c|c|c|c|}
\hline \multirow[b]{2}{*}{ No. } & \multirow{2}{*}{$\begin{array}{l}\text { Leaf rust resistance } \\
(L r) \text { genes }\end{array}$} & \multicolumn{14}{|c|}{ Infection types to $P t$ races } \\
\hline & & TGPQ & FHJQ & PHTS & KHSS & FGSS & KHSQ & FHNQ & KHJS & FHSQ & FHTQ & FGSD & FGGS & FGSR & PHJT \\
\hline 1 & RL6003 (Lrl) & 4 & 1 & 4 & $; 1$ & 1 & 0 & 1 & 1 & $; 1$ & 1 & $; 1$ & $; 1$ & $; 1$ & 3 \\
\hline 2 & RL6016 (Lr2a) & 4 & 2 & 2 & $3+$ & 0 & 3 & 2 & 3 & 1 & 1 & $; 1$ & ; & 2 & 1 \\
\hline 3 & RL6047 (Lr2C) & $3+$ & 4 & 4 & 4 & 4 & 4 & 4 & $3+$ & 4 & 3 & $3+$ & $3+$ & $3+$ & $3+$ \\
\hline 4 & RL6002 (Lr3) & 4 & 4 & 4 & 4 & 4 & 4 & 4 & 4 & 4 & 4 & $3+$ & $3+$ & $3+$ & $3+$ \\
\hline 5 & RL6010 (Lr9) & 0 & 0 & 0 & ; & 0 & 0 & 0 & 0 & 0 & 0 & 0 & 0 & 0 & 0 \\
\hline 6 & RL6005 (Lr16) & $3+$ & 4 & 4 & 4 & 4 & 4 & 4 & 4 & $3+$ & 4 & $3+$ & $3+$ & $3+$ & $3+$ \\
\hline 7 & RL6064 (Lr24) & 0 & 0 & 0 & 0 & 0 & 1 & 0 & 1 & 0 & 0 & $; 1$ & ; & $; 1$ & ; \\
\hline 8 & RL6078 (Lr26) & 1 & $3+$ & 3 & 3 & 2 & $3+$ & 3 & 3 & 3 & 3 & 2 & 1 & 2 & 3 \\
\hline 9 & RL6007 (Lr3ka) & 3 & 2 & 4 & 3 & 3 & 3 & 3 & 2 & $3+$ & 4 & 3 & 2 & 4 & 2 \\
\hline 10 & RL605 (Lr11) & 2 & 4 & 4 & 4 & $3+$ & 3 & 2 & 4 & 4 & $3+$ & $3+$ & 3 & $3+$ & $3+$ \\
\hline 11 & RL6008 (Lr17) & 3 & 3 & $3+$ & $3+$ & $3+$ & $3+$ & $3+$ & 3 & 3 & $3+$ & 3 & $2+$ & 3 & $3+$ \\
\hline 12 & RL6049 (Lr30) & 4 & 12 & 4 & ; & 1 & 2 & 12 & 1 & 2 & 3 & $1+$ & $1+$ & 1 & 2 \\
\hline 13 & RL6051 ( $L r B)$ & $3+$ & 4 & 4 & 4 & 4 & 4 & 4 & 4 & 4 & $3+$ & $3 \mathrm{C}$ & $3+$ & $3+$ & $3+$ \\
\hline 14 & RL6004 (Lr10) & 4 & 4 & 4 & 4 & 4 & 4 & 4 & 3 & $3+$ & 4 & $3 \mathrm{C}$ & 3 & $3+$ & 4 \\
\hline 15 & RL6013 (Lr14a) & 1 & 2 & 3 & 3 & $3+$ & 2 & $1+$ & 3 & 2 & 2 & $3+$ & 3 & 2 & 3 \\
\hline 16 & RL6009 (Lr18) & 1 & 1 & 0 & 2 & 0 & 0 & 1 & 2 & 2 & 2 & 1 & 2 & 3 & 3 \\
\hline 17 & RL6019 (Lr2b) & $3+$ & $3+$ & 4 & 4 & 2 & 2 & 3 & 3 & 3 & 2 & 2 & $3+$ & 2 & 2 \\
\hline 18 & RL6042 (Lr3bg) & $3+$ & 4 & 4 & 4 & 3 & 3 & $3+$ & 3 & 4 & 4 & 3 & 3 & $3+$ & $3+$ \\
\hline 19 & RL4031 (Lr13) & 4 & 4 & 4 & 3 & $3+$ & 3 & 4 & 4 & 4 & $3+$ & 3 & 3 & $3+$ & 3 \\
\hline 20 & RL6006 (Lr14b) & 3 & 4 & 4 & 4 & $2+$ & 4 & 4 & 3 & 4 & 4 & $3+$ & $3+$ & $3+$ & 4 \\
\hline 21 & RL6052 (Lr15) & 4 & 0 & 0 & 1 & 0 & 1 & 0 & 0 & 0 & 0 & ; & 0 & 0 & $3+$ \\
\hline 22 & RL6040 (Lr19) & ; & 0 & ; & 0 & 0 & ; & ; & 0 & 0 & 0 & ; & ; & 0 & 0 \\
\hline 23 & RL6092 (Lr20) & 3 & $3+$ & 4 & $3+$ & ; & 3 & 4 & 4 & ; & 2 & ; & ; & 1 & ; \\
\hline 24 & RL6043 (Lr21) & 3 & 4 & 2 & 3 & $2+$ & 3 & 2 & 2 & 3 & 3 & $1+$ & 2 & 2 & 3 \\
\hline 25 & RL6012 (Lr23) & $3+$ & 4 & 4 & 4 & $3+$ & 4 & 4 & 3 & $3+$ & 4 & 3 & $3+$ & $3+$ & $3+$ \\
\hline 26 & RL6079 (Lr28) & ; & 0 & ; & 0 & 0 & ; & ; & 1 & ; & 0 & 0 & ; & 0 & 0 \\
\hline 27 & RL6080 (Lr29) & 1 & 0 & 2 & 12 & 1 & 1 & 1 & 2 & 1 & 2 & ; & 2 & 2 & 1 \\
\hline 28 & RL6057 (Lr33) & 3 & 4 & 4 & 4 & 4 & 4 & 3 & 3 & 2 & 3 & $3 \mathrm{C}$ & 3 & $3+$ & 3 \\
\hline 29 & E84018 (Lr36) & 3 & 4 & 4 & $3+$ & 3 & 3 & $2+$ & 2 & 2 & 2 & $1+$ & $1+$ & 2 & 1 \\
\hline 30 & KS86NGRC02 (Lr39) & 4 & 4 & 4 & $3+$ & 4 & 4 & 4 & 3 & $3+$ & 3 & 3 & $3+$ & 3 & 3 \\
\hline 31 & KS91WGRC11 (Lr42) & 0 & 12 & 1 & $; 1$ & 0 & 1 & ; & 1 & 1 & 1 & ; & $1+$ & 1 & 2 \\
\hline 32 & RL6144 (Lr45) & 4 & 4 & 4 & $3+$ & 4 & 4 & 2 & 4 & 4 & $3+$ & 3 & 1 & 3 & $3+$ \\
\hline 33 & PAVON76 (Lr47) & 0 & 0 & 0 & 0 & 0 & 0 & 0 & 0 & 0 & ; & 0 & 0 & 0 & 0 \\
\hline 34 & C78.5 $(\operatorname{Lr} 51)$ & 0 & 0,1 & ; & 1 & ; & 1 & 1 & $; 1$ & 0 & ; & ; & ; & $; 1$ & ; \\
\hline
\end{tabular}

a According to the 0 to 4 Stakman scale as modified by Roelfs et al. (1992). 
data. Cultivars that showed high ITs to mixed $P t$ races (TGPQ, PHTS, and PHJT) at the seedling stage and had low MDS at the adult-plant stage (not significantly high MDS compared with the resistant control, Saar, in the field) were considered to have slow rusting resistance (Table 5).

\section{Results}

$L r$ genes based on gene postulation and molecular marker detection. A total of 34 differential lines, 50 wheat cultivars, and susceptible control (Zhengzhou 5389) were inoculated with 14
$P t$ races at seedling stage in the greenhouse (Tables 2 and 3). The differential lines for $11 \mathrm{Lr}$ genes $(\operatorname{Lr} 2 c, \operatorname{Lr} 3, \operatorname{Lr} 16, \operatorname{Lr} 17, \operatorname{Lr} B, \operatorname{Lr} 10$, $L r 3 b g, L r 13, L r 14 b, L r 23$, and $L r 39$ ) had high ITs to all $P t$ races. Low ITs to all $P t$ races were recorded for eight $L r$ genes $(L r 9$, Lr24, Lr19, Lr28, Lr29, Lr42, Lr47, and Lr51), which made it impossible to postulate these genes. However, 15 differential lines with Lr1, Lr2a, Lr26, Lr11, Lr3ka, Lr30, Lr14a, Lr18, Lr2b, Lr15, $L r 20, L r 21, L r 33, L r 36$, or $L r 45$ had different ITs when inoculated with different $P t$ races and, therefore, could be postulated for presence or absence in other wheat cultivars. The results of the gene

Table 3. Seedling infection types ${ }^{\mathrm{a}}$ and presence or absence of leaf rust resistance $(\mathrm{Lr})$ genes in 50 Ethiopian wheat cultivars based on gene postulation using 14 Puccinia triticina races and molecular markers

Infection types to $P t$ races

\begin{tabular}{|c|c|c|c|c|c|c|c|c|c|c|c|c|c|c|c|}
\hline Line no.b & $L r$ gene & TGPQ & FHJQ & PHTS & KHSS & FGSS & KHSQ & FHNQ & KHJS & FHSQ & FHTQ & FGSD & FGGS & FGSR & $\overline{\text { PHJT }}$ \\
\hline 1 & $\operatorname{Lr} 30^{\mathrm{c}}$ & 4 & 2 & $3+$ & 2 & 2 & 1 & 2 & 2 & 1 & $3+$ & 1 & 1 & 2 & 2 \\
\hline 2 & $\operatorname{Lr} 18^{\mathrm{c}}$ & 1 & 1 & 1 & 1 & ; & 1 & 1 & 1 & ; & 1 & 0 & 2 & 3 & 3 \\
\hline 3 & $\ldots$ & 2 & ; & ; & 0 & 2 & 1 & 0 & ; & ; & 1 & 2 & 3 & 1 & 0 \\
\hline 4 & $\ldots$ & 4 & 4 & 4 & 4 & 4 & 4 & 4 & 4 & 4 & 4 & 3 & 4 & 3 & 4 \\
\hline 5 & $L r 3 k a^{\mathrm{c}}$ & 3 & 2 & 4 & 3 & 3 & 4 & 3 & 1 & 4 & 3 & 3 & 1 & 4 & 2 \\
\hline 6 & $\operatorname{Lr} 15,^{\mathrm{c}} \operatorname{Lr} 46^{\mathrm{d}}$ & 3 & 1 & 0 & 0 & 1 & 0 & 2 & 1 & 1 & 1 & 1 & 2 & 2 & 4 \\
\hline 7 & $\operatorname{Lr} 26,{ }^{\mathrm{c}, \mathrm{d}} \operatorname{Lr} 46$ & 1 & $3+$ & 4 & 4 & 0 & 4 & 3 & 4 & 4 & 3 & 2 & 1 & 0 & 2 \\
\hline 8 & $\ldots$ & 2 & 2 & 4 & 2 & $2+$ & 3 & 1 & 1 & 1 & 2 & 3 & 2 & 3 & 1 \\
\hline 9 & $\operatorname{Lr} 26$ & 2 & 3 & 4 & 3 & 2 & 4 & 3 & 4 & 3 & 3 & 1 & 1 & 1 & 3 \\
\hline 10 & $\operatorname{Lr} 1^{\mathrm{c}, \mathrm{d}}$ & 3 & 1 & 4 & 1 & 0 & 1 & 1 & 0 & 1 & 1 & 1 & 1 & 2 & 3 \\
\hline 11 & Lr30, Lr46 & 3 & 1 & 4 & 2 & 2 & ; & 1 & ; & 1 & 3 & 2 & 2 & 1 & 1 \\
\hline 12 & $L r 1, L r 34^{\mathrm{d}}$ & 3 & 12 & 4 & $; 1$ & 12 & 12 & 1 & 1 & ; & 1 & 2 & 1 & 1 & 4 \\
\hline 13 & $\operatorname{Lrl}$ & 3 & $; 1$ & 4 & ; & 1 & $; 1$ & $; 1$ & 0 & 1 & 1 & 0 & 1 & 1 & $3+$ \\
\hline 14 & $\ldots$ & $; 1$ & 1 & 4 & 2 & ; & 1 & 0 & ; & 0 & ; & 3 & 2 & 2 & 1 \\
\hline 15 & $\ldots$ & 1 & 1 & 0 & 1 & ; & ; & ; & ; & 0 & 1 & 3 & 1 & 1 & 2 \\
\hline 16 & $\operatorname{Lr} 2 a^{\mathrm{c}}$ & 3 & 12 & 1 & 3 & 0 & $3+$ & 1 & 3 & 1 & 2 & 0 & 2 & 1 & 2 \\
\hline 17 & $\operatorname{Lr} 34, \operatorname{Lr} 68^{\mathrm{d}}$ & $; 1$ & 2 & 4 & 2 & 1 & 3 & 1 & $2+$ & 1 & 1 & 2 & 3 & 1 & 2 \\
\hline 18 & Lr3ka, Lr34 & $3+$ & 2 & 4 & 3 & $3+$ & 4 & 3 & 2 & 4 & 3 & 3 & 1 & 4 & 1 \\
\hline 19 & $L r 1$ & 3 & 0 & $3+$ & ; & 0 & ; & 0 & 0 & ; & ; & 2 & 1 & 1 & 4 \\
\hline 20 & $\ldots$ & 0 & 0 & 0 & 0 & 0 & 0 & 0 & 0 & ; & 0 & $2+$ & 1 & 2 & 1 \\
\hline 21 & $\operatorname{Lr} 18$ & ; & ; & ; & ; & 1 & 0 & 0 & 0 & ; & 0 & 0 & 1 & 4 & 3 \\
\hline 22 & Lr1, Lr34, Lr46 & 3 & 1 & 4 & $; 1$ & 1 & 1 & 1 & 0 & 1 & 1 & 0 & 2 & 1 & 3 \\
\hline 23 & Lr30, Lr34, Lr46 & 3 & 2 & 4 & ; & 2 & 1 & 1 & 1 & 2 & $3+$ & 2 & 1 & 1 & 1 \\
\hline 24 & $\operatorname{Lr} 18$ & $; 1$ & 1 & 2 & ; & 1 & 1 & 2 & 1 & 1 & 1 & 2 & 1 & 3 & $3+$ \\
\hline 25 & Lr26, Lr34 & 1 & 3 & 4 & 4 & 2 & 4 & 3 & $3+$ & 3 & 4 & 1 & 1 & 1 & 2 \\
\hline 26 & $\operatorname{Lr} 15, \operatorname{Lr} 34$ & 3 & 0 & 12 & 0 & 1 & 1 & 0 & ; & 0 & 1 & 0 & 2 & 0 & $3+$ \\
\hline 27 & $\operatorname{Lr} 1, \operatorname{Lr} 34$ & 4 & $; 1$ & 4 & ; & $; 1$ & 2 & 1 & ; & ; & 1 & 0 & 0 & 2 & 4 \\
\hline 28 & $L r 1, L r 46$ & 4 & 0 & 3 & $; 1$ & ; & ; & 1 & 0 & 1 & 1 & 1 & 0 & 2 & 4 \\
\hline 29 & $L r 2 a$ & $3+$ & 2 & 1 & $3+$ & 12 & 3 & 2 & $3+$ & 1 & 2 & 1 & 1 & 2 & 2 \\
\hline 30 & $\ldots$ & 0 & 0 & ; & ; & ; & ; & $; 1$ & 0 & ; & ; & 1 & 2 & 1 & 1 \\
\hline 31 & $\operatorname{Lrl} 18$ & $; 1$ & ; & 0 & 0 & ; & ; & 0 & 0 & 1 & 0 & 1 & 0 & 4 & $3+$ \\
\hline 32 & Lr30, Lr34, Lr46, Lr68 & 3 & 1 & 4 & 1 & 2 & 1 & 2 & 1 & 0 & 4 & 1 & 2 & 1 & 1 \\
\hline 33 & $\operatorname{Lr} 26$ & 2 & 4 & 4 & $3+$ & ; & 3 & 3 & 4 & 3 & 3 & 1 & 1 & 0 & 2 \\
\hline 34 & $\operatorname{Lr} 26$ & 1 & 3 & 4 & 3 & 2 & $3+$ & 3 & 3 & 4 & 3 & 2 & 0 & 2 & 1 \\
\hline 35 & Lr2a, Lr34 & $3+$ & 1 & 2 & $3+$ & 1 & 3 & 2 & 4 & ; & 1 & 2 & 1 & 2 & 2 \\
\hline 36 & $\ldots$ & 0 & 0 & ; & 0 & 0 & 0 & 0 & 0 & 0 & 0 & 2 & 0 & $2+$ & 0 \\
\hline 37 & $L r 1, L r 46$ & 3 & $; 1$ & 3 & $; 1$ & $; 1$ & ; & $; 1$ & $; 1$ & ; & 1 & 2 & 2 & 0 & 3 \\
\hline 38 & $\ldots$ & 1 & 12 & ; & 0 & 1 & 1 & 1 & ; & 1 & 1 & 4 & 1 & 1 & 3 \\
\hline 39 & $L r 1, L r 46$ & 3 & $; 1$ & 4 & ;2 & 1 & 1 & ; & ; & ; & 0 & 1 & 1 & 2 & 3 \\
\hline 40 & $L r 1, L r 46$ & 4 & ; & 4 & 0 & 1 & 0 & 1 & 0 & ; & 0 & 0 & 1 & 2 & 3 \\
\hline 41 & $\ldots$ & 0 & ; & ; & ; & 1 & ; & $; 1$ & ; & ; & ; & 1 & 2 & 2 & 1 \\
\hline 42 & $\operatorname{Lr} 20, \operatorname{Lr} 46$ & 4 & 3 & 4 & $3+$ & 1 & 3 & 4 & 3 & 0 & 1 & 1 & 2 & 1 & 1 \\
\hline 43 & $L r 3 k a$ & $3+$ & 12 & 4 & 3 & $3+$ & 4 & 4 & 2 & 3 & 4 & 3 & 1 & 3 & 2 \\
\hline 44 & Lr15, Lr34 & 3 & 2 & 1 & 2 & 12 & 1 & 1 & 1 & 1 & 1 & 0 & 2 & 1 & 4 \\
\hline 45 & Lrl & 3 & $; 1$ & 4 & ; & 01 & 1 & 1 & ; & 1 & 0 & 0 & 2 & 2 & 3 \\
\hline 46 & $\operatorname{Lr} 14 a^{\mathrm{c}}$ & 2 & 1 & 4 & 3 & 3 & 1 & 2 & 4 & 1 & 1 & 3 & 3 & 1 & 3 \\
\hline 47 & $L r 1, L r 46$ & 4 & 0 & 4 & $; 1$ & 1 & 0 & 0 & 1 & 1 & 0 & ; & 2 & 1 & 3 \\
\hline 48 & Lrl & 3 & 0 & 4 & 0 & 1 & ; & 1 & 1 & ; & 1 & 1 & ; & 0 & 3 \\
\hline 49 & $\operatorname{Lr11}{ }^{\mathrm{c}}, \operatorname{Lr} 34, \operatorname{Lr} 46$ & 2 & 3 & 4 & 3 & 3 & 4 & 1 & 3 & 3 & 3 & 3 & 3 & 4 & 3 \\
\hline 50 & $\ldots$ & 0 & ; & 0 & ; & ; & ; & $; 1$ & 0 & ; & 0 & 0 & 1 & 1 & 2 \\
\hline
\end{tabular}

a According to the 0 to 4 Stakman scale as modified by Roelfs et al. (1992).

b Line numbers correspond to those in Table 1.

c Postulation of $L r$ genes based on gene postulation.

d Detection of $\mathrm{Lr}$ genes based on molecular marker. 
postulation and molecular detection showed that $13 \mathrm{Lr}$ genes $(\mathrm{Lrl}$, Lr2a, Lr3ka, Lr11, Lr14a, Lr15, Lr18, Lr20, Lr26, Lr30, Lr34, $L r 46$, and $L r 68$ ), either singly or in combination, were identified in 39 cultivars. Ten cultivars (Biqa, Dashen, Galil, Gambo, Hogona, Lemu, Millennium, Monqolle, Shorima, and Wabe) contained unknown resistance genes because they displayed low ITs to most of the $P t$ races, whereas only Bobicho showed high ITs to all the tested $P t$ races.

Lrl was present in 13 cultivars. Eight cultivars (Enkoy, Hobora, Pavon-76, ET13A2, Sula, Dinknesh, Tsehay, and Kubsa) contained only $\operatorname{Lrl}$ because they were resistant to $11 \mathrm{Lrl}$ avirulent races and susceptible to three $P t$ races (Tables 3 and 4). Four cultivars (Ogalcho, Kulkulu, Mitike, and Tossa) contained Lrl combined with Lr46. K6295-4A contained Lr1, Lr34, and Lr46 in combination. All cultivars that carried $L r l$ based on gene postulation were confirmed by molecular marker detection.

Five cultivars were found to contain Lr26 (Tables 2 and 3). Digelu, Mekelle-02, and Mekelle-03 contained only Lr26. Katar and Dereselgn possessed $L r 26$ in combination with $L r 34$ and $L r 46$, respectively. The cultivars postulated to have $L r 26$ were also confirmed by the specific molecular marker for $L r 26$. Four cultivars (Abola, Dure, Kakaba, and Mekelle-01) were postulated to have Lr30. Only Lr30 was identified in Abola, because it was resistant to $11 \mathrm{Pt}$ races but susceptible to the remaining three $P t$ races (TGPQ, PHTS, and FHTQ). The other three cultivars were found to have Lr3O and Lr46 in combination.

Mekelle-04, Gassy, and Lakech contained Lr2a because they were found with low reaction to $10 L r 2 a$ avirulent $P t$ races (FHJQ, PHTS, FGSS, FHNQ, FHSQ, FHTQ, FGSD, FGGS, FGSR, and PHJT) (Tables 2 and 3). Only Lr3ka was postulated in Sirbo and Bolo, because they had low reactions to four $L r 3 k a$ avirulent $P t$ races (FHJQ,
KHJS, FGGS, and PHJT). Lr3ka in combination with Lr34 was detected in Hidasse. Kingbird and Suf-Omer contained Lr15 and Lr34 in combination. $L r 15$ and $L r 46$ were detected in Dandaa. These cultivars had low reaction to $12 \mathrm{Lr} 15$ avirulent races. $\mathrm{Lr} 18$, ineffective to $12 P t$ races but effective against only two $P t$ races (FGSR and PHJT), was postulated in Alidoro, Mandoyu, KBG-01, and Huluka. Lr20 in combination with $L r 46$ was detected only in Simba, because it was found with low ITs to seven $L r 20$ avirulent $P t$ races (FGSS, FHSQ, FHTQ, FGSD, FGGS, FGSR, and PHJT). Simba was also confirmed to contain $L r 20$ by the specific molecular marker for $L r 20$. Tusie was found to contain $L r 11, L r 34$, and $L r 46$ in combination, because it had low ITs to two $L r 11$ avirulent $P t$ races (TGPQ and FHNQ). Only $L r 14 a$ was postulated in Tay, because it was resistant to seven LrI $a$ avirulent races (TGPQ, FHJQ, KHSQ, FHNQ, FHSQ, FHTQ, and FGSR) (Tables 3 and 4).

Wheat cultivars that carried $L r 1, L r 20$, and $L r 26$ were confirmed by molecular marker detection and gene postulation. All cultivars lacked $\operatorname{Lr} 9, \operatorname{Lr} 19$, and $L r 24$ based on the molecular marker genotyping and seedling reaction. Postulation of $\operatorname{Lr} 34, \operatorname{Lr} 37, \operatorname{Lr} 46, \operatorname{Lr} 67$, and Lr68 was not possible in the seedling tests because all are APR genes. Therefore, the closely linked molecular markers with these APR genes were used to test all the cultivars. The results indicated that Lr46 and Lr34 were detected in 13 and 12 cultivars, respectively. Two cultivars, Hawi and Mekelle-01, were found with $\operatorname{Lr} 68$ and other known genes in combination. None of the cultivars contained Lr37 and Lr67.

Overall, the combined results of molecular marker genotyping and gene postulation showed that $13 \mathrm{Lr}$ genes $(\mathrm{Lrl}, \mathrm{Lr} 2 \mathrm{a}, \mathrm{Lr} 3 \mathrm{ka}, \mathrm{Lrl1}$, Lr14a, Lr15, Lr18, Lr20, Lr26, Lr30, Lr34, Lr46, and Lr68) either singly or in combination were identified in 39 cultivars, whereas none of the known $L r$ genes could be found in the remaining 11 cultivars.

Table 4. PCR primers and their conditions for detecting leaf rust resistance $(L r)$ genes as well as specific fragment sizes

\begin{tabular}{|c|c|c|c|c|c|c|c|}
\hline $\begin{array}{l}\text { Lr } \\
\text { gene }\end{array}$ & $\begin{array}{c}\text { Marker } \\
\text { type }^{\mathbf{a}}\end{array}$ & Primer & Sequence of primer $\left(5^{\prime} \rightarrow 3^{\prime}\right)$ & $\begin{array}{l}\text { Annealing } \\
\text { temp. }\left({ }^{\circ} \mathrm{C}\right)\end{array}$ & Size (bp) & Chr. & Reference \\
\hline Lrl & STS & $\begin{array}{l}\text { WR003F } \\
\text { WR003R }\end{array}$ & $\begin{array}{l}\text { GGGACAGAGACCTTGGTGGA } \\
\text { GAC GATGATGATTTGCTGCTGG }\end{array}$ & 65 & 760 & $5 \mathrm{DL}$ & Cloutier et al. (2007) \\
\hline $\operatorname{Lr} 9$ & STS & $\begin{array}{l}\mathrm{J} 13 / 1 \\
\mathrm{~J} 13 / 2\end{array}$ & $\begin{array}{l}\text { TCCTTTTATTCCGCACGCCGG } \\
\text { CCACACTACCCCAAAGAGAG }\end{array}$ & 66 & 1,100 & $6 \mathrm{BL}$ & Schachermayr et al. (1994) \\
\hline Lr10 & STS & $\begin{array}{l}\text { Lrk10D1 } \\
\text { Lrk10D2 }\end{array}$ & $\begin{array}{l}\text { GAAGCCCTTCGTCTCATCTG } \\
\text { TTGATTCATTGCAGATGAGATCACG }\end{array}$ & 58 & 282 & $1 \mathrm{AS}$ & Schachermayr et al. (1997) \\
\hline Lr19 & SCAR & $\begin{array}{l}\text { SCS265 F } \\
\text { SCS265 R }\end{array}$ & $\begin{array}{l}\text { GGCGGATAAGCAGAGCAGAG } \\
\text { GGCGGATAAGTGGGTTATGG }\end{array}$ & 65 & 512 & $7 \mathrm{DL}$ & Gupta et al. (2005) \\
\hline $\operatorname{Lr} 19$ & SCAR & $\begin{array}{l}\text { SCS253 F } \\
\text { SCS253 R }\end{array}$ & $\begin{array}{l}\text { GCTGGTTCCACAAAGCAAA } \\
\text { GGCTGGTTCCTTAGATAGGTG }\end{array}$ & 60 & 736 & $7 \mathrm{DL}$ & Gupta et al. (2005) \\
\hline $\operatorname{Lr} 20$ & STS & $\begin{array}{l}\text { STS638-L } \\
\text { STS638-R }\end{array}$ & $\begin{array}{l}\text { ACAGCGATGAAGCAATGAAA } \\
\text { GTCCAGTTGGTTGATGGAAT }\end{array}$ & 60 & 542 & $7 \mathrm{AL}$ & Neu et al. (2002) \\
\hline $\operatorname{Lr} 24$ & STS & $\begin{array}{l}\text { Lr24 J 9/1 } \\
\text { Lr24 J 9/2 }\end{array}$ & $\begin{array}{l}\text { TCTAGTCTGTACATGGGGGC } \\
\text { TGGCACATGAACTCCATACG }\end{array}$ & 57 & 310 & $6 \mathrm{BL}$ & Schachermayr et al. (1995) \\
\hline $\operatorname{Lr} 26$ & STS & $\begin{array}{l}\text { Glu-B3F } \\
\text { Glu-B3R }\end{array}$ & $\begin{array}{l}\text { GGTACCAACAACAACAACCC } \\
\text { GTTGCTGCTGAGGTTGGTTC }\end{array}$ & 65 & 210 & $1 \mathrm{BL}$ & Froidmont (1998) \\
\hline $\operatorname{Lr} 26$ & STS & $\begin{array}{l}\omega \text {-secalin F } \\
\omega \text {-secalin R }\end{array}$ & $\begin{array}{l}\text { ACC TTCCTCATCTTTGTCCT } \\
\text { CCGATGCCTATACCACTACT }\end{array}$ & 65 & 1,076 & $1 \mathrm{BL}$ & Chai et al. (2006) \\
\hline $\operatorname{Lr} 34$ & CAPS & $\begin{array}{l}\text { cssfr6-f } \\
\text { cssfr6 -r }\end{array}$ & $\begin{array}{l}\text { CTGAGGCACTCTTTCCTGTACAAAG } \\
\text { GCATTCAATGAGCAATGGTTATC }\end{array}$ & 67.5 & 135 and 451 & 7DS & Lagudah et al. (2009) \\
\hline Lr37 & STS & $\begin{array}{l}\text { VENTRIUP } \\
\text { LN2 }\end{array}$ & $\begin{array}{l}\text { AGGGGCTACTGACCAAGGCT } \\
\text { TGCAGCTACAGCAGTATGTACACAAAA }\end{array}$ & 65 & 259 & $2 \mathrm{AS}$ & Bariana and McIntosh (1993) \\
\hline$L r 46$ & CAPS & $\begin{array}{l}\operatorname{csLV} 46 G 22 F \\
\operatorname{csLV} 46 G 22 R\end{array}$ & $\begin{array}{l}\text { AGGGAAAAGACATCTTTTTTTTC } \\
\text { CGACCGACTTCGGGTTC }\end{array}$ & 58 & 335 & $1 \mathrm{BL}$ & Lagudah, E. S. (pers. comm.) \\
\hline Lr46 & SSR & Xwmc44 & $\begin{array}{l}\text { TCTTCTGGGCTTTGATCCTG } \\
\text { TGTTGCTAGGGACCCGTAGTGG }\end{array}$ & 61 & 242 & $1 \mathrm{BL}$ & Lillemo et al. (2008) \\
\hline $\operatorname{Lr67}$ & SSR & $X c f d 23-4 D$ & $\begin{array}{l}\text { TAGCAGTAGCAGCAGCAGGA } \\
\text { GCAAGGAAGAGTGTTCAGCC }\end{array}$ & 60 & 187 & 4DL & Herrera-Foessel et al. (2011) \\
\hline $\operatorname{Lr67}$ & SSR & $\begin{array}{l}C F D 71-F \\
C F D 71-R\end{array}$ & $\begin{array}{l}\text { CAATAAGTAGGCCGGGACAA } \\
\text { TGTGCCAGTTGAGTTTGCTC }\end{array}$ & 60 & 216 & $4 \mathrm{DL}$ & Herrera-Foessel et al. (2011) \\
\hline $\operatorname{Lr68}$ & STS & $\begin{array}{l}c s G S-F 1 \\
c s G S-R 1\end{array}$ & $\begin{array}{l}\text { AAGATTGTTCACAGATCCATGTCA } \\
\text { GAGTATTCCGGCTCAAAAAGG }\end{array}$ & 60 & 385 & $7 \mathrm{BL}$ & Herrera-Foessel et al. (2012) \\
\hline
\end{tabular}

a STS = sequence-tagged site; SCAR = sequence characterized amplified region; CAPS = cleaved amplified polymorphic sequence; and SSR = simple sequence repeat. 
APR to leaf rust. The analysis of variance results for MDS of the tested cultivars indicated that there were significant differences among cultivars, environments, and cultivar-environment interactions in the field $(P<0.05)$ (Table 5). This revealed that cultivar and cultivar-environment interactions influenced the APR for leaf rust. However, no significant differences were observed between replications and cultivar-replication interactions, as expected. The MDS values of Zhengzhou 5389 ranged from 80 to $95 \%$, and that of Saar was $5 \%$, at all environments, demonstrating adequate levels of leaf rust disease (Table 6). MDS values of all the cultivars including the resistant control, Saar, and susceptible control, Zhengzhou 5389, are presented in Supplementary Table S1. Twenty-one cultivars with high ITs to the mixed Pt races at the seedling stage but showing low MDS values in the field across all the environments were considered to have slow rusting resistance.

\section{Discussion}

Postulation of seedling-effective $L r$ genes performed based on the gene-for-gene hypothesis (Flor 1956) enables quick identification of genes present in wheat cultivars with diverse genetic backgrounds.

Lrl was first described in Malakoff (Mains et al. 1926) and reported as Lrl in 1946 (Ausemus et al. 1946). Cloutier et al. (2007) mapped Lrl on chromosome 5DL. Mebrate et al. (2008) reported four cultivars with $\mathrm{Lrl}$ among a collection of 23 wheat cultivars from Ethiopia tested with $31 \mathrm{Pt}$ races. In the present study, $L r l$ was the most commonly identified seedling $L r$ gene among the tested 50 cultivars (Table 3). In the same way reported by Singh and Rajaran (1991), Pavon-76 was found to contain Lrl. According to its pedigree analysis, $\mathrm{Lrl}$ in Pavon-76 and Kubsa might be derived from Vicam-71 (Singh 1993) and Bluebird (McIntosh et al. 1995), respectively. Lr26, originated from rye (Secale cereal L.), exists in wheat in the 1BL/1RS translocation. According to Rajaram et al. (1983), the 1BL/1RS translocation region also carried $\mathrm{Sr} 31, \mathrm{Yr} 9$, and $\mathrm{Pm} 8$ genes for resistance to stem rust, stripe rust, and powdery mildew, respectively, and served as a basis of resistance genes used in wheat breeding. In this study, $L r 26$ was identified in five cultivars (Table 3). $L r 26$ in Digelu might be originated from Kauz or Mex according to the pedigree analysis (Singh and Rajaran 1991). Lr30, as a seedling recessive resistance gene originated from Terenzio, was mapped on chromosome 4AL (Dyck and Kerber 1981). Lr30 was identified in four cultivars (Table 3). Similar to the present study, Mebrate et al. (2008) also reported Lr30 in Abola. McIntosh and Baker (1968) cytologically mapped $\mathrm{Lr} 15$ closely linked with $\mathrm{Sr} 6$ on chromosome 2DS. Lr15 is widely effective when combined with an APR gene like Lr34 (Dholakia et al. 2013). In the present study, three cultivars contained Lr15 in combination with other APR genes (Table 3). Based on the pedigree analysis, Lr15 in Suf-Omer might be from Frontana (Bansal 1988).

Dyck $(1977,1987)$ characterized $L r 34$ from the very beginning in Canada; however, $\operatorname{Lr} 34$ had been used in the expansion of new wheat cultivars from the early 20th century. Lr34 was the first cloned slow rusting resistance gene that has remained durable for more than 50 years (Krattinger et al. 2009; Lagudah et al. 2009). Cloning of Lr34 enabled a gene-specific DNA marker to be developed (Dakouri et al. 2010; Lagudah et al. 2009), which provides a powerful tool for marker assisted selection (MAS) in wheat breeding. In this study, 12 cultivars were detected with Lr34 (Table 3). The presence of Lr34 in Hawi, Kakaba, and Kubsa might be derived from Parula (McIntosh et al. 2012), Rayon-89 (Singh 1993), and Bluebird (McIntosh et al. 1995), respectively, based on the pedigree analysis. Lr34 in Enkoy and Suf-Omer might be derived from Frontana (McCallum et al. 2012), and that of Katar and Tusie might be from Cook (McIntosh et al. 1995).

According to William et al. (2003), Lr46 has been found widely in wheat genotypes originating from CIMMYT. Lr46 was originally identified from Pavon-76 on chromosome 1BL (Singh et al. 1998). Xwmc44 marker, linked to Lr46 (Lillemo et al. 2008), is being used in MAS. Although Lr46 has a similar phenotype to Lr34, resistance to multiple pathogens (Lillemo et al. 2008; William et al. 2003), the resistance conferred by $L r 46$ is not as obvious as $\operatorname{Lr34}$ (Kolmer et al. 2018a; Lagudah 2011). In the present study, Lr46 was found in 13 cultivars. According to the pedigree analysis, Lr46 in Hawi, Simba, and Tossa might be derived from Rayon-89 (Kolmer et al. 2008), Parula (McIntosh et al. 2012), and Attila (McIntosh et al. 2008), respectively.

Lr68 is a slow rusting resistance gene mapped on chromosome 7BL (Herrera-Foessel et al. 2012). This gene originated from Frontana, which also possessed Lr34 and Lr46. The dominant marker $\operatorname{cs} G S$ was afterward developed for $\operatorname{Lr} 68$ (Herrera-Foessel et al. 2012). Lr68 in Hawi might be derived from Parula (McIntosh et al. 2012).

Table 5. Analysis of variance of maximum disease severity in 50 wheat genotypes, including slow rusting cultivar Saar and susceptible Zhengzhou 5389 checks, tested in the 2017-2018 and 2018-2019 growing seasons ${ }^{\mathrm{a}}$

\begin{tabular}{lrccr}
\hline Source of variation & df & Mean square & $\boldsymbol{F}$ value & \multicolumn{1}{c}{$\boldsymbol{P}$} \\
\hline Cultivar & 51 & $1,310.39$ & $67.03 *$ & $<0.0001$ \\
Environment & 2 & $1,072.69$ & $54.87 *$ & $<0.0001$ \\
Replication & 1 & $3,459.73$ & 176.97 & 1.9624 \\
Cultivar $\times$ environment & 102 & 125.49 & $6.42 *$ & $<0.0001$ \\
Cultivar $\times$ replication & 51 & 72.27 & 3.70 & 2.8647 \\
Error & 104 & 19.55 & & \\
\hline
\end{tabular}

a $*$ = significant at 0.05 level of probability.

Table 6. Seedling infection types (ITs) ${ }^{\mathrm{a}}$ with mixed Puccinia triticina (Pt) races in the seedling test and maximum disease severity (MDS, \%) in field experiments in Baoding and Zhoukou in the 2017-2018 and 2018-2019 growing seasons for Ethiopian wheat cultivars with slow rusting resistance to leaf rust

\begin{tabular}{|c|c|c|c|c|c|}
\hline Line no. ${ }^{b}$ & $\begin{array}{l}\text { APRc } \\
\text { genes }\end{array}$ & $\begin{array}{l}\text { Seedling } \\
\text { ITs to } \\
\text { mixed } \boldsymbol{P t} \\
\text { races }\end{array}$ & $\begin{array}{c}\text { 2017-2018 } \\
\text { Baoding } \\
\text { MDS }(\%)\end{array}$ & $\begin{array}{l}\text { 2018-2019 } \\
\text { Baoding } \\
\text { MDS (\%) }\end{array}$ & $\begin{array}{c}\text { 2018-2019 } \\
\text { Zhoukou } \\
\text { MDS }(\%)\end{array}$ \\
\hline 1 & $\ldots$ & 4 & 5 & 5 & 5 \\
\hline 2 & $\ldots$ & 4 & 0 & 5 & 0 \\
\hline 6 & Lr46 & 3 & 1 & 5 & 0 \\
\hline 8 & $\ldots$ & 4 & 5 & 5 & 5 \\
\hline 11 & Lr46 & 3 & 0 & 10 & 5 \\
\hline 14 & $\ldots$ & 3 & 1 & 5 & 5 \\
\hline 16 & $\ldots$ & 4 & 1 & 5 & 1 \\
\hline 17 & Lr34 & $3+$ & 5 & 5 & 1 \\
\hline 21 & $\ldots$ & 4 & 5 & 15 & 0 \\
\hline 25 & Lr34 & $3-$ & 0 & 5 & 0 \\
\hline 27 & Lr34 & $3+$ & 1 & 5 & 1 \\
\hline 28 & Lr46 & 3 & 1 & 5 & 0 \\
\hline 35 & Lr34 & 3 & 0 & 5 & 1 \\
\hline 37 & Lr46 & 4 & 0 & 5 & 0 \\
\hline 38 & $\ldots$ & $3+$ & 1 & 5 & 0 \\
\hline 39 & Lr46 & 3 & 5 & 1 & 5 \\
\hline 40 & $L r 46$ & 4 & 10 & 1 & 5 \\
\hline 42 & $L r 46$ & 4 & 1 & 5 & 5 \\
\hline 47 & Lr46 & 4 & 1 & 5 & 5 \\
\hline 48 & $\ldots$ & 4 & 1 & 10 & 0 \\
\hline 49 & $\begin{array}{l}\text { Lr34, } \\
\text { Lr46 }\end{array}$ & 4 & 0 & 1 & 0 \\
\hline Saar $^{d}$ & & 3 & 5 & 5 & 5 \\
\hline $\begin{array}{l}\text { Zhengzhou } \\
5389^{\mathrm{e}}\end{array}$ & & 4 & 80 & 95 & 90 \\
\hline $\operatorname{LSD}^{\mathrm{f}}$ & & & & 5.06 & \\
\hline
\end{tabular}

a According to the 0 to 4 Stakman scale as modified by Roelfs et al. (1992).

${ }^{\mathrm{b}}$ Line numbers correspond to those in Table 1.

c Adult plant resistance genes based on molecular marker.

d Slow rusting check.

e Susceptible check.

${ }^{\mathrm{f}} \mathrm{LSD}=$ least significant difference. 
The seedling $L r$ genes in 11 cultivars out of the tested 50 cultivars could not be identified. It might be better to use different $P t$ races with various pathogenicity or additional differential lines with other resistance genes to postulate the genes in these cultivars.

Even though the seedling $L r$ genes were postulated based on the gene-for-gene approach, there was an observable limitation to this strategy. It was not able to identify all the $L r$ genes in the tested cultivars due to restrictions of virulence in $P t$ races. It is possible that five cultivars (Hogona, Lemu, Millennium, Shorima, and Wabe) might harbor unknown major resistance genes to leaf rust, because they were detected with low ITs to all the tested $P t$ races at the seedling stage in the greenhouse and showed resistant response to the mixed $P t$ races used in the field nurseries at the adult-plant stage. Genotyping the cultivars using molecular markers linked to the $\mathrm{Lr}$ genes was more effective than gene postulation. Identification of Lr1, Lr20, Lr26, Lr34, Lr46, and Lr68 using molecular markers was reliable. Hence, the molecular markers linked with these $L r$ genes might be very useful in MAS for developing wheat cultivars for resistance to leaf rust.

Singh et al. (2000) stated that APR has proven to be durable, having been widely deployed in CIMMYT spring wheat cultivars. Twenty-five $(50 \%)$ of the tested cultivars originated from CIMMYT genotypes, and APR genes were widely deployed in them (Tables 1 and 3). $L r 34, L r 46$, and $\operatorname{Lr} 68$ either singly or in combination with other genes were detected in 21 cultivars. The remaining 10 cultivars might have unknown APR genes. The mixed $P t$ races were virulent to most of these cultivars at the seedling stage, and the APR in these cultivars might be obtained from $L r 34, L r 46, L r 68$, and other unknown APR genes.

In summary, the gene postulation and molecular marker detection results revealed $13 \mathrm{Lr}$ genes $(\mathrm{Lr} 1, \mathrm{Lr} 2 a, \mathrm{Lr} 3 \mathrm{ka}, \mathrm{Lr} 11, \mathrm{Lr} 14 a, \mathrm{Lr} 15$, Lr18, Lr20, Lr26, Lr30, Lr34, Lr46, and Lr68) either singly or in combination in 39 cultivars. Ten cultivars might contain undetectable $\mathrm{Lr}$ genes, whereas only one cultivar, Bobicho, may contain no effective $L r$ gene. Additional $P t$ races with various pathogenicity or using more differential lines with other resistance genes might be better to postulate the genes in these cultivars. Twenty-one cultivars were found with slow rusting resistance. $\operatorname{Lr} 34, \operatorname{Lr} 46$, and $\operatorname{Lr} 68$, either singly or in combination with other genes, were detected in 21 cultivars. The results should be valuable for selecting cultivars to grow and used in breeding new cultivars with improved resistance to leaf rust in Ethiopia and China.

\section{Literature Cited}

Ausemus, E. R., Harrington, J. B., Reitz, L. P., and Worzella, W. W. 1946. A summary of genetic studies in hexaploid and tetraploid wheats. J. Am. Soc. Agron. 38:1082-1099.

Badebo, A., Eshetu, B., Berhanu, B., Bekele, H., and Melaku, D. 2008. Review of two decades of research on diseases of small cereal crops. Pages 375-429 in: Proceedings of the 14th Annual Conference of the Plant Protection Society of Ethiopia (PPSE), December 19-22, 2006, Addis Ababa, Ethiopia.

Bansal, V. K. 1988. Lr genes, morphological characters and peroxidase isozymes of wheat (Triticum aestivum L.) cv. Frontana. Cereal Res. Commun. 16: 203-209.

Bariana, H. S., and McIntosh, R. A. 1993. Cytogenetic studies in wheat. XV. Location of rust resistance genes in VPM1 and their genetic linkage with other disease resistance genes in chromosome 2A. Genome 36:476-482.

Caldwell, R. M. 1968. Breeding for general and/or specific plant disease resistance. Pages 263-272 in: Proceedings of the 3rd International Wheat Genetics Symposium. K. W. Findlay and K. W. Shepherd, eds. Australian Academy of Science, Canberra, Australia.

Central Statistical Agency (CSA). 2017. Agricultural Sample Survey 2016/17 (2009 E.C.), Volume I, Report on Area and Production of Major Crops (Private Peasant Holdings, Meher Season). Statistical Bulletin 584. The Federal Democratic Republic of Ethiopia, Addis Ababa, Ethiopia.

Chai, J. F., Zhou, R. H., Jia, J. Z., and Liu, X. 2006. Development and application of a new codominant PCR marker for detecting 1BL.1RS wheat-rye chromosome translocations. Plant Breed. 125:302-304.

Chen, X. M. 2013. High-temperature adult-plant resistance, key for sustainable control of stripe rust. Am. J. Plant Sci. 4:608-627.

Chen, X. M. 2014. Integration of cultivar resistance and fungicide application for control of wheat stripe rust. Can. J. Plant Pathol. 36:311-326.

Cloutier, S., McCallum, B. D., Loutre, C., Banks, T. W., Wicker, T., Feuillet, C., Keller, B., and Jordan, M. C. 2007. Leaf rust resistance gene $L r 1$, isolated from bread wheat (Triticum aestivum L.) is a member of the large psr567 gene family. Plant Mol. Biol. 65:93-106.

Cobb, J. 1948. Outline for the study of scoliosis. Instr. Course Lect. 5:261-275.

Dakouri, A., McCallum, B. D., Walichnowski, A. Z., and Cloutier, S. 2010. Finemapping of the leaf rust Lr34 locus in Triticum aestivum (L.) and characterization of large germplasm collections support the $\mathrm{ABC}$ transporter as essential for gene function. Theor. Appl. Genet. 121:373-384.

Dholakia, B. B., Rajwade, A. V., Hosmani, P., Khan, R. R., Chavan, S., Reddy, D. M. R., Lagu, M. D., Bansal, U. K., Saini, R. G., and Gupta, V. S. 2013. Molecular mapping of leaf rust resistance gene $\mathrm{Lrl5}$ in hexaploid wheat. Mol. Breed. 31:743-747.

Dubin, H. J., Johnson, R., and Stubbs, R. W. 1989. Postulated genes for resistance to stripe rust in selected CIMMYT and related wheats. Plant Dis. 73:472-475.

Dyck, P. L. 1977. Genetics of leaf rust reaction in three introductions of common wheat. Can. J. Genet. Cytol. 19:711-716.

Dyck, P. L. 1987. The association of a gene for leaf rust resistance with the chromosome 7D suppressor of stem rust resistance in common wheat Genome 29:467-469.

Dyck, P. L., and Kerber, E. R. 1981. Aneuploid analysis of a gene for leaf rust resistance derived from the common wheat cultivar Terenzio. Can. J. Genet. Cytol. 23:405-409

Flor, H. H. 1942. Inheritance of pathogenicity in Melampsora lini. Phytopathology 32:653-669.

Flor, H. H. 1956. The complementary genic systems in flax and flax rust. Adv Genet. 8:29-54.

Froidmont, D. D. 1998. A co-dominant marker for the 1BL/1RS wheat-rye translocation via multiplex PCR. J. Cereal Sci. 27:229-232.

Gao, P., Zhou, Y., Gebrewahid, T. W., Zhang, P., Yan, X., Li, X., Yao, Z., Li, Z., and Liu, D. 2019. Identification of known leaf rust resistance genes in common wheat cultivars from Sichuan province in China. Crop Prot. 115:122-129.

Gebrewahid, T. W., Yao, Z., Yan, X. C., Gao, P., and Li, Z. 2017. Identification of leaf rust resistance genes in Chinese common wheat cultivars. Plant Dis. 101: 1729-1737.

Gill, K. S., Lubbers, E. L., Gill, B. S., Raupp, W. J., and Cox, T. S. 1991. A genetic linkage map of Triticum tauschii (DD) and its relationship to the D genome of bread wheat (AABBDD). Genome 34:362-374.

Gupta, S. K., Charpe, A., Koul, S., Prabhu, K. V., and Haque, Q. M. 2005 Development and validation of molecular markers linked to an Aegilops umbellulata-derived leaf-rust-resistance gene, $\operatorname{Lr} 9$, for marker-assisted selection in bread wheat. Genome 48:823-830.

Hei, N. B. 2017. Evaluation of wheat cultivars for slow rusting resistance to leaf rust (Puccinia trticina Eriks) in Ethiopia. Afr. J. Plant Sci. 11:23-29.

Helguera, M., Khan, I. A., Kolmer, J., Lijavetzky, D., Zhong-qi, L., and Dubcovsky, J. 2003. PCR assays for the Lr37-Yr17-Sr38 cluster of rust resistance genes and their use to develop isogenic hard red spring wheat lines. Crop Sci. 43:1839-1847.

Herrera-Foessel, S. A., Laguda, E. S., Huerta-Espino, J., Hayden, M. J., Bariana, H. S., Singh, D., and Singh, R. P. 2011. New slow-rusting leaf rust and stripe rust resistance genes $\operatorname{Lr67}$ and $\mathrm{Yr} 46$ in wheat are pleiotropic or closely linked. Theor. Appl. Genet. 122:239-249.

Herrera-Foessel, S. A., Singh, R. P., Huerta-Espino, J., Rosewarne, G. M., Periyannan, S. K., Viccars, L., Calvo-Salazar, V., Lan, C. X., and Lagudah, E. S. 2012. Lr68: A new gene conferring slow rusting resistance to leaf rust in wheat. Theor. Appl. Genet. 124:1475-1486.

Kolmer, J. A., Bernardo, A., Bai, G., Hayden, M. J., and Chao, S. 2018a. Adult plant leaf rust resistance derived from Toropi wheat is conditioned by $\operatorname{Lr} 78$ and three minor QTL. Phytopathology 108:246-253.

Kolmer, J. A., Singh, R. P., Garvin, D. F., Viccars, L., William, H. M., HuertaEspino, J., Ogbonnaya, F. C., Raman, H., Orford, S., Bariana, H. S., and Lagudah, E. S. 2008. Analysis of the $\mathrm{Lr} 34 / \mathrm{Yr} 18$ rust resistance region in wheat germplasm. Crop Sci. 48:1841-1852.

Krattinger, S. G., Lagudah, E. S., Spielmeyer, W., Singh, R. P., Huerta-Espino, J., McFadden, H., Bossolini, E., Selter, L. L., and Keller, B. 2009. A putative ABC transporter confers durable resistance to multiple fungal pathogens in wheat. Science 323:1360-1363.

Lagudah, E. 2011. Molecular genetics of race non-specific rust resistance in wheat Euphytica 179:81-91.

Lagudah, E. S., Krattinger, S. G., Herrera-Foessel, S., Singh, R. P., HuertaEspino, J., Spielmeyer, W., Brown-Guedira, G., Selter, L. L., and Keller, B. 2009. Gene-specific markers for the wheat gene $\mathrm{Lr} 34 / \mathrm{Yr} 18 / \mathrm{Pm} 38$ which confers resistance to multiple fungal pathogens. Theor. Appl. Genet. 119 889-898.

Lagudah, E. S., McFadden, H., Singh, R. P., Huerta-Espino, J., Bariana, H. S., and Spielmeyer, W. 2006. Molecular genetic characterization of the $\mathrm{Lr34/Yr18}$ slow rusting resistance gene region in wheat. Theor. Appl. Genet. 114:21-30.

Li, Z. F., Xia, X. C., He, Z. H., Li, X., Zhang, L. J., Wang, H. X., Meng, Q. F., Yang, W. X., Li, G. Q., and Liu, D. Q. 2010. Seedling and slow rusting resistance to leaf rust in Chinese wheat cultivars. Plant Dis. 94:45-53.

Lillemo, M., Asalf, B., Singh, R. P., Huerta-Espino, J., Chen, X. M., He, Z. H., and Bjørnstad, А. 2008. The adult plant rust resistance loci $\mathrm{Lr34/Yr18}$ and $\mathrm{Lr} 46 /$ Yr29 are important determinants of partial resistance to powdery mildew in bread wheat line Saar. Theor. Appl. Genet. 116:1155-1166. 
Long, D. L., and Kolmer, J. A. 1989. A North American system of nomenclature for Puccinia recondita f. sp. tritici. Phytopathology 79:525-529.

Maaroof, E. M., Singh, R. P., Julio, H. E., and Atiq, R. 2005. Resistance of some Iraqi bread wheat cultivars to Puccinia triticina. Phytopathol. Mediterr. 44: 247-255.

Mains, E. B., Leighty, C. E., and Johnston, C. O. 1926. Inheritance of resistance to leaf rust Puccinia triticina Erikss., in crosses of common wheat, Triticum vulgare Vill. J. Agric. Res. 32:931-972.

McCallum, B. D., Humphreys, D. G., Somers, D. J., Dakouri, A., and Cloutier, S. 2012. Allelic variation for the rust resistance gene $L r 34 / Y r 18$ in Canadian wheat cultivars. Euphytica 183:261-274.

McIntosh, R. A., and Baker, E. P. 1968. A linkage map for chromosome 2D. Pages 305-308 in: Proceedings of the 3rd International Wheat Genetics Symposium. K. W. Finlay and K. W. Shepard, eds. Australian Academy of Science, Sydney, Australia.

McIntosh, R. A., Dubcovsky, J., and Rogers, W. J. 2012. Catalogue of gene symbols for wheat: 2012 supplement. Annu. Wheat Newsl. 58:72-74.

McIntosh, R. A., Dubcovsky, J., Rogers, W. J., Morris, C., and Xia, X. C. 2017. Catalogue of gene symbols for wheat: 2017 supplement. [2017-3-21]. https:// shigen.nig.ac.jp/wheat/komugi/genes/macgene/supplement2017.pdf.

McIntosh, R. A., Wellings, C. R., and Park, R. F. 1995. Wheat Rust: An Atlas of Resistance Genes. CSIRO, Canberra, Australia.

McIntosh, R. A., Yamazaki, Y., Dubcovsky, J., Rogers, J., Morris, C., Somers, D. J., Appels, R., and Devos, K. M. 2008. Catalogue of gene symbols for wheat. 11th International Wheat Genetics Symposium, 24-29 August 2008, Brisbane, Australia.

Mebrate, S. A., Dehne, H. W., Pillen, K., and Oerke, E. C. 2008. Postulation of seedling leaf rust resistance genes in selected Ethiopian and German bread wheat cultivars. Crop Sci. 48:507-516.

Neu, C., Stein, N., and Keller, B. 2002. Genetic mapping of the Lr20-PmI resistance locus reveals suppressed recombination on chromosome arm 7AL in hexaploid wheat. Genome 45:737-744.

Pathan, A. K., and Park, R. F. 2006. Evaluation of seedling and adult plant resistance to leaf rust in European wheat cultivars. Euphytica 149:327-342.
Rajaram, S., Mann, C. H. E., Ortiz-Ferrara, G., and Mujeeb-Kazi, A. 1983. Adaptation, stability and high yield potential of certain 1B/1R CIMMYT wheats. Pages 613-621 in: Proceedings of the 6th International Wheat Genetics Symposium. S. Sakamoto, ed. Kyoto University, Japan.

Roelfs, A. P., Singh, R. P., and Saari, E. E. 1992. Rust Diseases of Wheat Concepts and Methods of Disease Management. Centro Internacional de Mejoramientode Maiz y Trigo, Mexico City, Mexico.

Schachermayr, G., Feuillet, C., and Keller, B. 1997. Molecular markers for the detection of the wheat leaf rust resistance gene $\mathrm{LrlO}$ in diverse genetic backgrounds. Mol. Breed. 3:65-74.

Schachermayr, G., Siedler, H., Gale, M. D., Winzeler, H., Winzeler, M., and Keller, B. 1994. Identification and localization of molecular markers linked to the $\operatorname{Lr} 9$ leaf rust resistance gene of wheat. Theor. Appl. Genet. 88: $110-115$.

Schachermayr, G. M., Messmer, M. M., Feuillet, C., Winzeler, H., Winzeler, M., and Keller, B. 1995. Identification of molecular markers linked to the Agropyron elongatum-derived leaf rust resistance gene $\mathrm{Lr} 24$ in wheat. Theor. Appl. Genet. 90:982-990.

Singh, R. P. 1993. Resistance to leaf rust in 26 Mexican wheat cultivars. Crop Sci. 33:633-637.

Singh, R. P., Chen, W. Q., and He, Z. H. 1999. Leaf rust resistance of spring, facultative, and winter wheat cultivars from China. Plant Dis. 83:644-651.

Singh, R. P., Huerta-Espino, J., and Rajaram, S. 2000. Achieving near-immunity to leaf and stripe rusts in wheat by combining slow rusting resistance genes. Acta Phytopathol. Entomol. Hung. 35:133-139.

Singh, R. P., Mujeeb-Kazi, A., and Huerta-Espino, J. 1998. Lr46: A gene conferring slow-rusting resistance to leaf rust in wheat. Phytopathology 88 : 890-894.

Singh, R. P., and Rajaran, S. 1991. Resistance to Puccinia recondita f. sp. tritici in 50 Mexican bread wheat cultivars. Crop Sci. 31:1472-1479.

William, M., Singh, R. P., Huerta-Espino, J., Islas, S. O., and Hoisington, D. 2003. Molecular marker mapping of leaf rust resistance gene Lr46 and its association with stripe rust resistance gene $Y r 29$ in wheat. Phytopathology 93:153-159. 